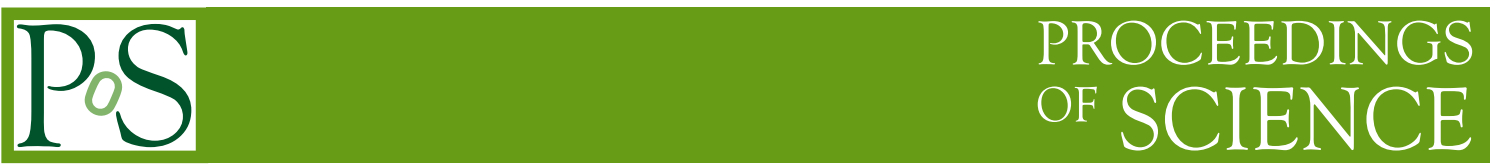

\title{
Decoding the nature of Dark Matter
}

\author{
Alexander Belyaev* \\ School of Physics and Astronomy, University of Southampton, Southampton SO17 1BJ, UK \\ E-mail: a.belyaevesoton.ac.uk
}

\begin{abstract}
Determination of the nature of Dark Matter (DM) is one of the most fundamental problems of particle physics and cosmology. If DM is light enough and interacts with Standard Model particles directly or via some mediators with a strength beyond the gravitational one, it can be directly produced at particle accelerators. The study of the complete set of dimension 5 and 6 effective DM operators which we present here demonstrates the LHC potential to distinguish operators with different spin of DM: they have a different energy dependence and respectively different distributions of the invariant mass of the DM pair which consequently leads to different missing transverse energy distributions. This study can be directly applied beyond the EFT paradigm, as we demonstrate for three cases - Supersymmetry (DM with spin 1/2), inert two Higgs doublet model (i2HDM) (spin 0 of DM) and minimal isotriplet of vector dark matter model (MVDM). We also stress an importance of complementarity of the collider searches, DM direct detection experiments as well as relic density and CMB data. For several appealing DM models as an example, we show that the interplay between high and low energy data has unique power for an identification of DM nature. We also highlight prospects of new signature from DM theories such as disappearing charge tracks which are characteristic for wide class of DM theories. Finally, we advocate the importance of the framework which would combine the experience of HEP community and would allow to effectively identify the underlying theory of DM.
\end{abstract}

Corfu Summer Institute 2018 "School and Workshops on Elementary Particle Physics and Gravity"

(CORFU2018)

31 August - 28 September, 2018

Corfu, Greece

${ }^{*}$ Speaker. 


\section{Introduction}

Understanding the nature of Dark Matter (DM) is one of the greatest puzzles of modern particle physics and cosmology. Although overwhelming observational evidences from galactic to cosmological scales point to the existence of DM [1, 2, 3], after decades of experimental effort only its gravitational interaction has been experimentally confirmed. Currently, no information is available on the DM properties, such as its spin, mass, interactions other than gravitational, symmetry responsible for its stability, number of states associated to it, and possible particles that would mediate the interactions between DM and the standard model (SM) particles.

If DM is light enough and interacts with SM particles directly or via some mediators with a strength beyond the gravitational one, its elusive nature can be detected or constrained in different ways: a) from direct production at colliders, resulting in a signature exhibiting an observed SM object, such as jet, Higgs, $Z$, or photon, that recoils against the missing energy from the DM pair [4, 5, 6, 7]; b) via the relic density constraint obtained through the observations of cosmic microwave background (CMB) anisotropies, such as those of WMAP and PLANCK collaborations [8, 1];c) from DM direct detection (DD) experiments, which are sensitive to elastic spin independent (SI) or spin dependent (SD) DM scattering off nuclei $[9,10,11,12]$; d) from DM indirect detection searches, that look for SM particles produced in the decay or annihilation of DM present in the cosmos, both with high energies observables (gamma-rays, neutrinos, charge cosmic rays) produced in the local Universe $[13,14,15,16,17,18]$, and by studying the effects of energy produced by DM annihilation in the early universe on the properties of the CMB spectrum [19, 20,1].

It is clear that decoding of the nature of
DM requires the respective signal at least in one of the search experiment. We do not have one. However even without having this signal at the moment we can already conclude on what kind of DM models are excluded already. Moreover, by exploring different signatures of one particular model, their correlation and interplay we can prepare ourselves to discovery of DM and their identification.

\section{Contact interactions}

In Table 1 we have summarised a minimal set of independent dimension-5 and dimension6 operators for complex scalar, Dirac fermion and complex vector DM coupling to quarks and gluons, adopting the widely used notations of $[21,22,23]$. These operators provide monojetsignature, the shapes of $\boldsymbol{E}_{\mathrm{T}}^{\text {miss }}$ distributions for which is presented in in Fig. 1 from Ref. [23] for DM mass of $10 \mathrm{GeV}$. One can observe a big difference in $\boldsymbol{E}_{\mathrm{T}}^{\text {miss }}$ shapes of the groups of the operators, primarily split into groups of operators with scalar, femion and vector DM. The origin of the different $\boldsymbol{E}_{\mathrm{T}}^{\text {miss }}$ shapes from different operators can be related to a combination of effects. First, for a fixed Lorentz structure of the SM part of the EFT operators, the same invariant mass distribution of the DM pair, $M_{\text {inv }}(D M, D M)$, uniquely defines the shape of the $\boldsymbol{E}_{\mathrm{T}}^{\text {miss }}$ distribution. Moreover, with the increase of $M_{\mathrm{inv}}(D M, D M)$, the $\boldsymbol{E}_{\mathrm{T}}^{\text {miss }}$ shape falls less and less steeply (again, for a given SM component of the EFT operator).

The reason why the bigger invariant mass of DM is correlated with flatter $\boldsymbol{E}_{\mathrm{T}}^{\mathrm{miss}}$ behaviour can be explained by the phase space and parton density effects [23]: when $M_{\text {inv }}(D M, D M)$ is small, the radiation of a high $P_{T}$ jet will "cost" a large relative shift in $x$, the transferred momentum of the parton, leading to a rapidly falling $\boldsymbol{E}_{\mathrm{T}}^{\text {miss }}$ distribution; on the contrary, when $M_{\mathrm{inv}}(D M, D M)$ is large, the radiation of a high $P_{T}$ jet will "cost" a small relative shift in $x$, which will lead to a more slowly falling $\boldsymbol{E}_{\mathrm{T}}^{\text {miss }}$ 
distribution in comparison to the first case.

\begin{tabular}{|c|c|}
\hline \multicolumn{2}{|l|}{ Complex Scalar DM } \\
\hline$\frac{g_{*}^{2}}{\Lambda} \phi^{\dagger} \phi \bar{q} q$ & {$[C 1]$} \\
\hline$\frac{g_{*}^{2}}{\Lambda} \phi^{\dagger} \phi \bar{q} i \gamma^{5} q$ & {$[C 2]$} \\
\hline$\frac{g_{*}^{2}}{\Lambda^{2}} \phi^{\dagger} i \overleftrightarrow{\partial_{\mu}} \phi \bar{q} \gamma^{\mu} q$ & {$[C 3]$} \\
\hline$\frac{g_{*}^{2}}{\Lambda^{2}} \phi^{\dagger} i \overleftrightarrow{\partial_{\mu}} \phi \bar{q} \gamma^{\mu} \gamma^{5} q$ & {$[C 4]$} \\
\hline$\frac{g_{*}^{2}}{\Lambda^{2}} \phi^{\dagger} \phi G^{\mu v} G_{\mu v}$ & {$[C 5]$} \\
\hline$\frac{g_{*}^{2}}{\Lambda^{2}} \phi^{\dagger} \phi \tilde{G}^{\mu v} G_{\mu \nu}$ & {$[C 6]$} \\
\hline \multicolumn{2}{|l|}{ Dirac Fermion DM } \\
\hline$\frac{g_{*}^{2}}{\Lambda^{2}} \bar{\chi} \chi \bar{q} q$ & {$[\mathrm{D} 1]$} \\
\hline$\frac{g_{*}^{2}}{\Lambda^{2}} \bar{\chi} i \gamma^{5} \chi \bar{q} q$ & {$[\mathrm{D} 2]$} \\
\hline$\frac{g_{*}^{2}}{\Lambda^{2}} \bar{\chi} \chi \bar{q} i \gamma^{5} q$ & [D3] \\
\hline$\frac{g_{*}^{2}}{\Lambda^{2}} \bar{\chi} \gamma^{5} \chi \bar{q} \gamma^{5} q$ & {$[\mathrm{D} 4]$} \\
\hline$\frac{g_{*}^{2}}{\Lambda^{2}} \bar{\chi} \gamma^{\mu} \chi \bar{q} \gamma_{\mu} q$ & {$[\mathrm{D} 5]$} \\
\hline$\frac{g_{*}^{2}}{\Lambda^{2}} \bar{\chi} \gamma^{\mu} \gamma^{5} \chi \bar{q} \gamma_{\mu} q$ & [D6] \\
\hline$\frac{g_{*}^{2}}{\Lambda^{2}} \bar{\chi} \gamma^{\mu} \chi \bar{q} \gamma_{\mu} \gamma^{5} q$ & [D7] \\
\hline$\frac{g_{*}^{2}}{\Lambda^{2}} \bar{\chi} \gamma^{\mu} \gamma^{5} \chi \bar{q} \gamma_{\mu} \gamma^{5} q$ & [D8] \\
\hline$\frac{g_{*}^{2}}{\Lambda^{2}} \bar{\chi} \sigma^{\mu v} \chi \bar{q} \sigma_{\mu v} q$ & [D9] \\
\hline$\frac{g_{*}^{2}}{\Lambda^{2}} \bar{\chi} \sigma^{\mu v} i \gamma^{5} \chi \bar{q} \sigma_{\mu \nu} q$ & {$[\mathrm{D} 10]$} \\
\hline \multicolumn{2}{|l|}{ Complex Vector DM } \\
\hline$\frac{g_{*}^{2} m_{\mathrm{DM}}^{2}}{\Lambda^{3}} V_{\mu}^{\dagger} V^{\mu} \bar{q} q$ & {$[\mathrm{~V} 1]$} \\
\hline$\frac{g_{*}^{2} m_{\mathrm{DM}}^{2}}{\Lambda^{3}} V_{\mu}^{\dagger} V^{\mu} \bar{q} i \gamma^{5} q$ & {$[\mathrm{~V} 2]$} \\
\hline$\frac{g_{*}^{2} m_{\mathrm{DM}}^{2}}{2 \Lambda^{4}} i\left(V_{v}^{\dagger} \partial_{\mu} V^{v}-V^{v} \partial_{\mu} V_{v}^{\dagger}\right) \bar{q} \gamma^{\mu} q$ & {$[\mathrm{~V} 3]$} \\
\hline$\frac{g_{*}^{2} m_{D M}^{2}}{2 \Lambda_{2}^{4}}\left(V_{v}^{\dagger} \partial_{\mu} V^{v}-V^{v} \partial_{\mu} V_{v}^{\dagger}\right) \bar{q} i \gamma^{\mu} \gamma^{5} q$ & {$[\mathrm{~V} 4]$} \\
\hline$\frac{g_{*}^{2} m_{\mathrm{DM}}^{2}}{\Lambda^{3}} V_{\mu}^{\dagger} V_{v} \bar{q} i \sigma^{\mu v} q$ & {$[\mathrm{~V} 5]$} \\
\hline$\frac{g_{*}^{2} m_{\mathrm{DM}}^{2}}{\Lambda^{3}} V_{\mu}^{\dagger} V_{v} \bar{q} \sigma^{\mu v} \gamma^{5} q$ & {$[\mathrm{~V} 6]$} \\
\hline$\frac{g_{*}^{2} m_{\mathrm{DM}}}{2 \Lambda^{3}}\left(V_{v}^{\dagger} \partial^{v} V_{\mu}+V_{v} \partial^{v} V_{\mu}^{\dagger}\right) \bar{q} \gamma^{\mu} q$ & {$[\mathrm{~V} 7 \mathrm{P}]$} \\
\hline$\frac{g_{*}^{2} m_{\mathrm{DM}}^{2}}{2 \Lambda^{4}}\left(V_{v}^{\dagger} \partial^{v} V_{\mu}-V_{v} \partial^{v} V_{\mu}^{\dagger}\right) \bar{q} i \gamma^{\mu} q$ & {$[\mathrm{~V} 7 \mathrm{M}]$} \\
\hline$\frac{g_{*}^{2} m_{\mathrm{DM}}}{2 \Lambda^{3}}\left(V_{v}^{\dagger} \partial^{v} V_{\mu}+V_{v} \partial^{v} V_{\mu}^{\dagger}\right) \bar{q} \gamma^{\mu} \gamma^{5} q$ & {$[\mathrm{~V} 8 \mathrm{P}]$} \\
\hline$\frac{g_{*}^{2} m_{D \mathrm{DM}}^{2}}{2 \Lambda^{4}}\left(V_{v}^{\dagger} \partial^{v} V_{\mu}-V_{v} \partial^{v} V_{\mu}^{\dagger}\right) \bar{q} i \gamma^{\mu} \gamma^{5} q$ & {$[\mathrm{~V} 8 \mathrm{M}]$} \\
\hline$\frac{g_{*}^{2} m_{\mathrm{DM}}}{2 \Lambda^{3}} \varepsilon^{\mu v \rho \sigma}\left(V_{v}^{\dagger} \partial_{\rho} V_{\sigma}+V_{v} \partial_{\rho} V_{\sigma}^{\dagger}\right) \bar{q} \gamma_{\mu} q$ & {$[\mathrm{~V} 9 \mathrm{P}]$} \\
\hline $0.5 \frac{g_{*}^{2} m_{\mathrm{DM}}}{2 \Lambda^{3}} \varepsilon^{\mu v \rho \sigma}\left(V_{v}^{\dagger} \partial_{\rho} V_{\sigma}-V_{v} \partial_{\rho} V_{\sigma}^{\dagger}\right) \bar{q} i \gamma_{\mu} q$ & {$[\mathrm{~V} 9 \mathrm{M}]$} \\
\hline$\frac{g_{*}^{2} m_{\mathrm{DM}}}{2 \Lambda^{3}} \varepsilon^{\mu v \rho \sigma}\left(V_{v}^{\dagger} \partial_{\rho} V_{\sigma}+V_{v} \partial_{\rho} V_{\sigma}^{\dagger}\right) \bar{q} \gamma_{\mu} \gamma^{5} q$ & {$[\mathrm{~V} 10 \mathrm{P}]$} \\
\hline$\frac{g_{*}^{2} m_{\mathrm{DM}}}{2 \Lambda^{3}} \varepsilon^{\mu \nu \rho \sigma}\left(V_{v}^{\dagger} \partial_{\rho} V_{\sigma}-V_{v} \partial_{\rho} V_{\sigma}^{\dagger}\right) \bar{q} i \gamma_{\mu} \gamma^{5} q$ & {$[\mathrm{~V} 10 \mathrm{M}]$} \\
\hline$\frac{g_{*}^{2} m_{\mathrm{DM}}^{2}}{\Lambda^{4}} V_{\mu}^{\dagger} V^{\mu} G^{\rho \sigma} G_{\rho \sigma}$ & {$[V 11]$} \\
\hline$\frac{g_{*}^{2} m_{\mathrm{DM}}^{2}}{\Lambda^{4}} V_{\mu}^{\dagger} V^{\mu} \tilde{G}^{\rho \sigma} G_{\rho \sigma}$ & {$[V 12]$} \\
\hline
\end{tabular}

Table 1: Minimal basis of operators of dimension six or less involving only complex scalar DM $(\phi)$, Dirac fermion DM $(\chi)$ or complex vector DM $\left(V^{\mu}\right)$ interacting with SM quarks $(q)$ or gluons. Here we denote the field strength tensor of the gluons as $G^{\mu v}$ and its dual as $\tilde{G}^{\mu v}$.

Different oprators have differentt energy behaviour and respective different invariant mass distributions. Majority scalar DM operators have the smallest mean value of $M_{\mathrm{inv}}(D M, D M)$, while vector $\mathrm{DM}$ has the largest mean value of $M_{\mathrm{inv}}(D M, D M)$ as demonstrated in In Figure 2. Because of relation of the shape of $M_{\text {inv }}(D M, D M)$ and $\boldsymbol{E}_{\mathrm{T}}^{\text {miss }}$ slope distributions one can distinguish many operators and related underlying theories between ear other by the shape of the $\boldsymbol{E}_{\mathrm{T}}^{\mathrm{miss}}$ signal: C1-C2,C5-C6,D9-D10,V1$\mathrm{V} 2, \mathrm{~V} 3-\mathrm{V} 4, \mathrm{~V} 5-\mathrm{V} 6$ and V11-12 pairs among each other [23] (given thatt that the signal is observed).

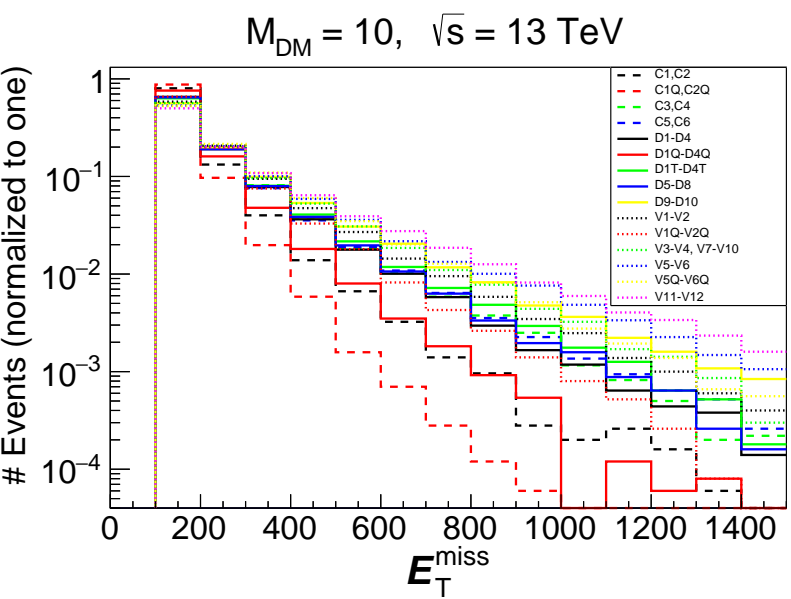

Figure 1: $\boldsymbol{E}_{\mathrm{T}}^{\mathrm{miss}}$ parton level distributions for a representative subset of the EFT operators from Table 1 for $13 \mathrm{TeV}$ LHC energy and $M_{\mathrm{DM}}=10 \mathrm{GeV}$. 


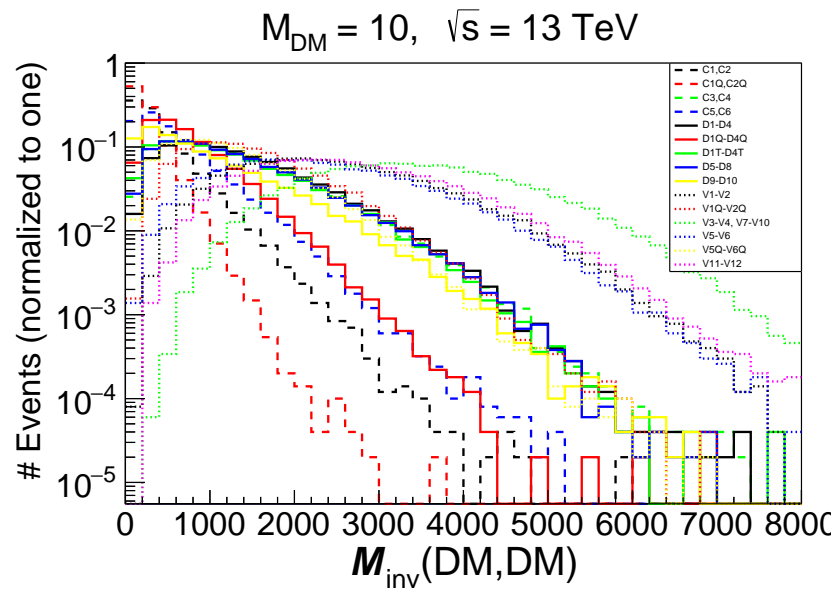

Figure 2: Invariant mass of DM pair distributions normalised to unity for EFT operators in Table 1 for $13 \mathrm{TeV}$ LHC energy, $M_{\mathrm{DM}}=10 \mathrm{GeV}$ and $p_{T, j e t} \geq 100 \mathrm{GeV}$ cut applied.

Non-collider DM searches play an important complementary role in probing DM parameter space. As an example in Fig. 3 (top) we present the non-collider constraints for the operators D2, which exhibit pseudoscalar interactions of fermion Dirac DM with quarks. One can see that even for momentum-suppressed operator D2 (because of its pseudo-scalar nature) DM DD constraints from Xenon[24] play an important role which is comparable to collider constraints, presented in Fig. 3 (bottom). It is important to stress that both LHC and DM DD searches set an upper limit on value of $\Lambda$. The LHC limit is of the order of $1 \mathrm{TeV}$ for present LHC data while DM DD searches the limit strongly depend on the operator. For example for non-suppressed operators conserving parity the limit on $\Lambda$ is about 3 orders of magnitude above the LHC one. On the other hand LHC limit is beyond DM DD searches for operators with suppressed elastic scattering cross sections on the nuclei (C2,C4,C6,D2,D3,D4,D6-10,V2,V4-V10).

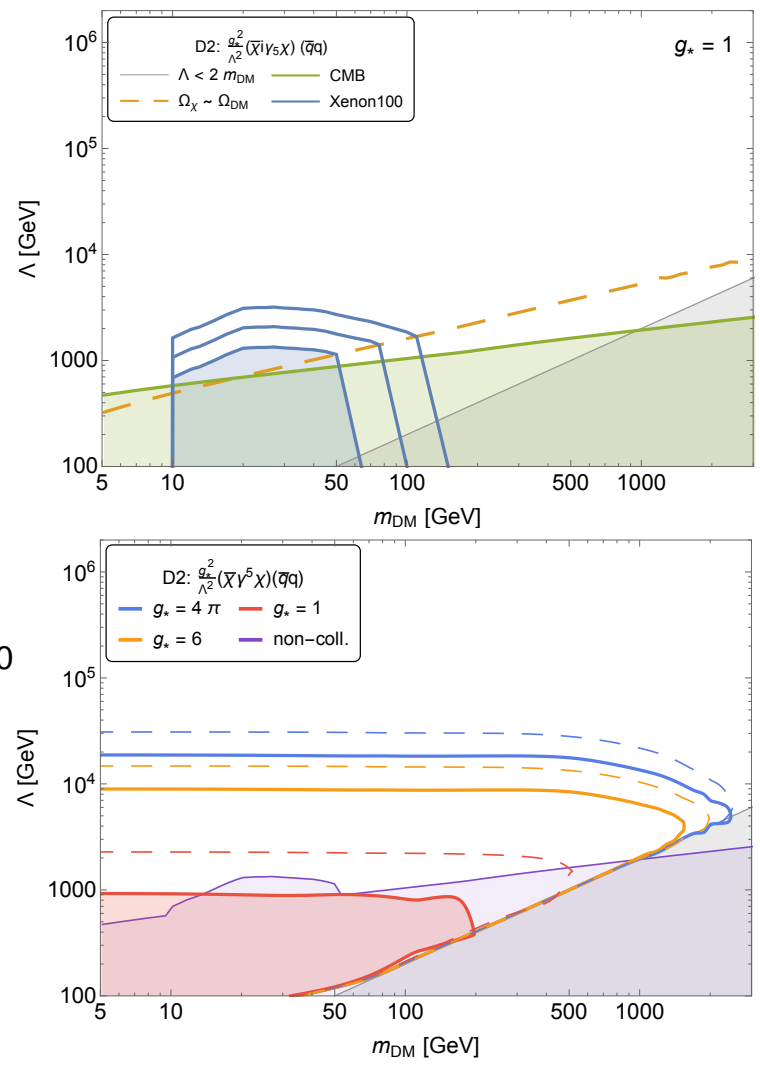

Figure 3: Top: Non-collider constraints on D2 operator with fermion DM: (i) SI DM DD searches (shaded blue region below the lowest blue contour), (ii) constraints from relic density (above the yellow dashed line), (iii) constraints from the CMB ( shaded green area) and (iv) constraints from the validity of the EFT $\left(\Lambda>2 m_{D M}\right)$. Bottom: LHC monojet constraints on D2 EFT operator. The area inside the red, orange and blue solid curves is excluded by current LHC data at $95 \%$ CL for $g_{\star}=1$, 6 and $4 \pi$, respectively. The projected LHC limits for $300 \mathrm{fb}^{-1}$ are indicated by dashed thin lines. The combined exclusion regions from CMB and DM DD searches for $g_{\star}=1$ are given by the lightpurple area. See details and complete set of plots in Ref [25].

Moreover for operators with pseudovector currents which have suppressed DM DD rates, one should take into account effect of their running from TeV energy scale at the LHC down to low energy scale at DM DD experiments, due to which an operator acquire non-negligible vector component [26, 27, 28]. 


\section{Beyond EFT}

The analysis of $\boldsymbol{E}_{\mathrm{T}}^{\mathrm{miss}}$ shape presented here can be applied to different scenarios, beyond the EFT approach in general, where the DM mediator is not produced on-the-mass-shell, such as the case of t-channel mediator or mediators with mass below $2 M_{D M}$, where the $M_{\text {inv }}(D M, D M)$ is not fixed. This case covers a wide range of theories. As an example in Fig. ?? the normalised shape for $\boldsymbol{E}_{\mathrm{T}}^{\mathrm{miss}}$ distribution from $p p \rightarrow \chi_{1}^{+} \chi_{1}^{-} / \chi_{1}^{ \pm} \chi_{1}^{0} \rightarrow$ $\chi_{1}^{0} \chi_{1}^{0}+$ softletons/jets Minimal Supersymmetric Model(MSSM) signal and its dominant irreducible background $Z+j e t \rightarrow v \bar{v}+$ jet $(Z j$, is presented for LHC@13TeV [29]. One car see that $\boldsymbol{E}_{\mathrm{T}}^{\text {miss }}$ distribution from the $\mathrm{SM} Z+j e$ background falls steeply than that from MSSM signal. this is related to the fact that invarian mass of $\chi_{1}^{0} \chi_{1}^{0}$ pair is larger than the mass of Z-boson.

Another example is given in Fig. 5 where we present $\boldsymbol{E}_{\mathrm{T}}^{\text {miss }}$ from $h_{1} h_{2} j$ inert two Higg، doublet model (i2HDM) signal alongside the estimated (by CMS) experimental backgrounc for $\sqrt{s}=13 \mathrm{TeV}$. Again, $\boldsymbol{E}_{\mathrm{T}}^{\text {miss }}$ distribution from SM BG falls more rapidly because the mean value of invariant mass of DM is bigger then the $Z$-boson mass.

Finally in Fig. ?? we present shapes of $p_{T}$ distributions for pair production of the charged component of the vector isotriplet from MVDM model [30] and of a wino from MSSM model, for different values of the masses. One can see that in case of MVDM model the $p_{T}$ distribution is flatter than in the MSSM case. This difference is again related to the mean value of invariant mass of odd particles distributions $\left(V^{+}\right.$and $\left.\chi^{+}\right)$respectively which has large mean value in case of MVDM model. This is an important feature since one can use MSSM results for MVDM reinterpretation using conservative estimation since the efficiency of the $p_{T}$ cuts will be higher for the
MVDM model.

An important feature of the $\boldsymbol{E}_{\mathrm{T}}^{\text {miss }}$ or $p_{T}$ signal shapes for these different DM theories is that has the same explanation as for EFT study case above - it is related to the invariant mass of the invisible (or charged in case of MVDM model) system. This feature provides a very important way to increase signalto-background ratio (S/B) (which is typically below $1 \%$ for low $\boldsymbol{E}_{\mathrm{T}}^{\text {miss }}$ cuts) by increasing the value of $\boldsymbol{E}_{\mathrm{T}}^{\text {miss }}$ or by performing the signalbackground shape analysis [31].

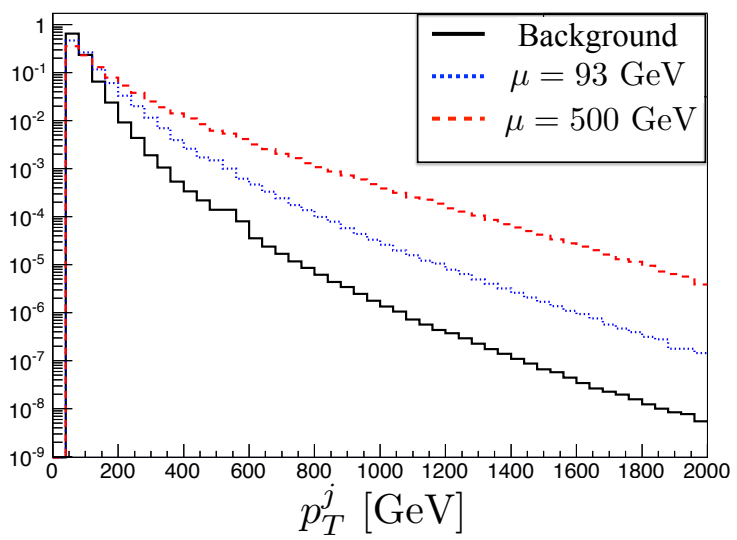

Figure 4: Signal (dotted blue and dashed red) and $Z j$ background (solid black) parton-level $p_{T}^{j}$ distributions for the $13 \mathrm{TeV}$ LHC for the NSUSY scenario: normalised signal and $Z j$ background distributions. See details in Ref [29].

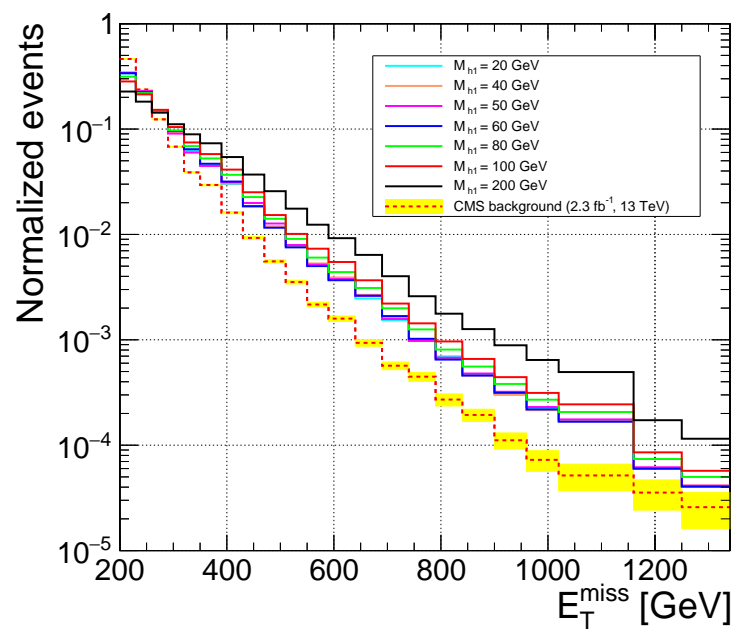

Figure 5: $\boldsymbol{E}_{\mathrm{T}}^{\mathrm{miss}}$ from $h_{1} h_{2} j$ i2HDM signal vs back- 
ground for $\sqrt{s}=13 \mathrm{TeV}$. See details in [31]

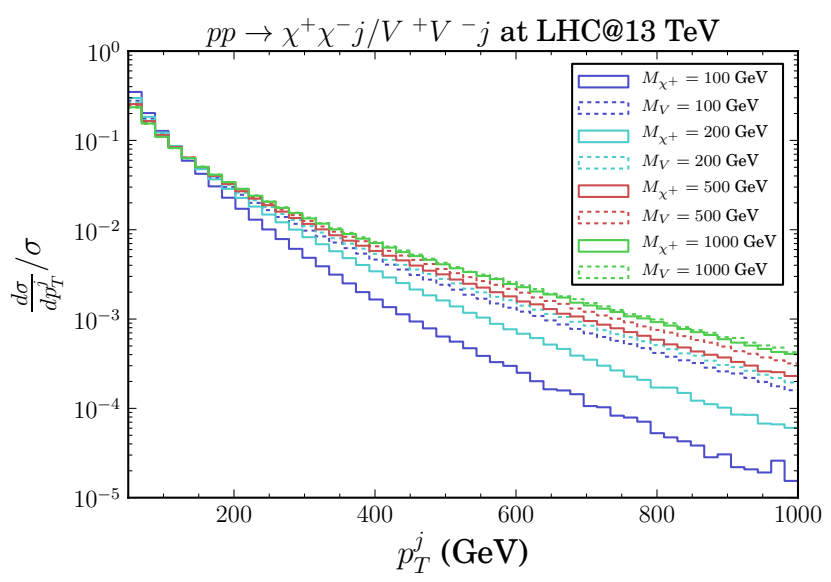

Figure 6: The shapes of $p_{T}$ distributions for pair production of the charged component of the vector isotriplet from MVDM model and of a wino from MSSM model, for different values of the masses. See details in [30]

The role of non-collider DM searches is also crucial in case of these two complete and consistent models. And an example in Fig. 7 we present the projected LHC reach for MSSM monojet signal in the $\Delta M=$ $m_{\chi_{1}^{+}}-m_{c h i_{1}^{0}}, M_{D M}=m_{c h i_{1}^{0}}$ parameter space together with LUX anx Xenon1T DM DD exclusion Ref [29]. One can see that LHC would be able cover neutralino DM mass only below 250 $\mathrm{GeV}$ (with the assumption that S/B of the order of $3 \%$ will be under control) even with $3 \mathrm{ab}^{-1}$ total integrated luminocity. It is worth to stress though that LHC will cover the region inaccessible by Xenon1T in small $\Delta M$ region, while Xenon1T is able to cover $m_{D M}$ well beyond the LHC reach for $\Delta M>3-5 \mathrm{GeV}$, demonstrating a very important complementarity of DM DD to collider searches of DM. In case of i2HDM model collider sensitivity with mono-jet signature is even more limited because of the lower production rates of the scalar DM, $h_{1}$, or its inert partners $\left(h_{2}\right.$ and $\left.h^{+}\right)$and expected LHC reach is below $100 \mathrm{GeV}$ for $M_{h 1}$.

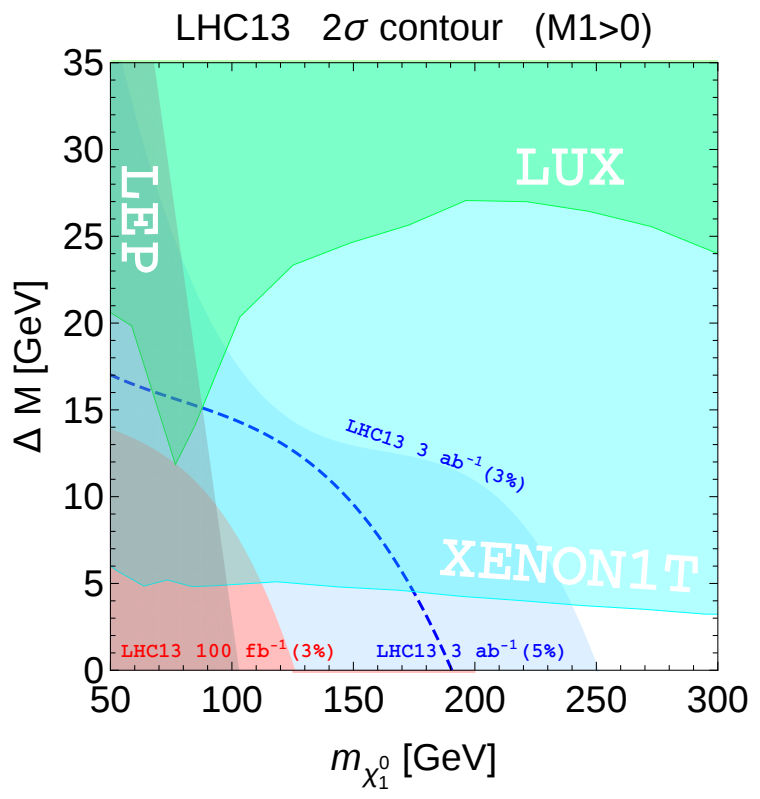

Figure 7: Exclusion contour lines for the $13 \mathrm{TeV}$ LHC at the end of the LHC Run2 (light red region) and of the HL-LHC (light blue region). The region excluded by LUX and Xenon1T are also shown, together with the LEP limit. See details in Ref [29].

\section{Beyond mono-X signature}

While mono- $X$ (with $X$ being jet, $\gamma, Z, H, t$ etc.) DM signatures at colliders are the most general ones, their rates is typically very low (usually at the percent level or even lower). Besides several others interesting but modelspecific DM signature studies one should stress one signature which can be also considered as quite generic one. In case when $\mathrm{DM}, D^{0}$, is embedded into electroweak multiplet and its mass split from the charged odd particle(s), $D^{+}$, is generated only radiatively (preserving gauge invariance), the one can find that the value of this mass split is of the order of $0.2 \mathrm{GeV}$. In this case $D^{+}$has a very small width and respectively large life-time driven by its dominant decay to DM and pion: $D^{+} \rightarrow D^{0} \pi^{0}$ which makes $D^{+}$long lived particles (LLP). Production of $D^{+}$in pairs or in association with DM leads then to the typical signature from charged LLP: disappearing charged tack (DCT) as soon as the 
track from LLP is long enough (from few $\mathrm{cm}$ to a meeter). In case of such signature the S/B ratio is much higher than in case of mono-jet signal and therefore, substantially bigger DM masses can be probed with charged LLPs from DM sector $[32,33,30]$. As an example, we would like to present here results for the minimal vector triplet DM $\left(V^{0}\right)$ model [30] which predicts the right amount of DM for $M_{D M}$ in the 3-4 TeV range depending on DM coupling tot he Higgs boson.

In this model the SM is supplemented by a new massive vector boson $V_{\mu}$ in the adjoint representation of $S U(2)_{L}$, e.g. by two new massive vector particles: $V^{0}$ and $V^{ \pm}$. If $V_{\mu}$ transforms homogeneously (i.e. $V_{\mu} \rightarrow g_{L}^{\dagger} V_{\mu} g_{L}$ where $\left.g_{L} \in S U(2)_{L}\right)$ and $Z_{2}$ symmetry is imposed (which links the quartic $V$ coupling to the gauge coupling constant and makes theory unitary is unitary before EW symmetry breaking and in the absence of the Higgs boson as found in Ref. [34]) then Lagrangian can be written as:

$$
\begin{aligned}
\mathscr{L} & =\mathscr{L}_{S M}-\operatorname{Tr}\left\{D_{\mu} V_{v} D^{\mu} V^{v}\right\} \\
& +\operatorname{Tr}\left\{D_{\mu} V_{v} D^{v} V^{\mu}\right\} \\
& -\frac{g^{2}}{2} \operatorname{Tr}\left\{\left[V_{\mu}, V_{v}\right]\left[V^{\mu}, V^{v}\right]\right\} \\
& -i g \operatorname{Tr}\left\{W_{\mu v}\left[V^{\mu}, V^{v}\right]\right\}+\tilde{M}^{2} \operatorname{Tr}\left\{V_{v} V^{v}\right\} \\
& +a\left(\Phi^{\dagger} \Phi\right) \operatorname{Tr}\left\{V_{v} V^{v}\right\}
\end{aligned}
$$

where $D_{\mu}=\partial_{\mu}-i g\left[W_{\mu},\right]$ is the usual $S U(2)_{L}$ covariant derivative in the adjoint representation and $\mathscr{L}_{S M}$ represents the SM Lagrangian. The main difference with respect to the model in Ref. [34] is that the $S U(2)_{L}$ symmetry is broken by the Higgs mechanism and the associated gauge bosons have mass. We thus allow for a coupling of $V$ to the Higgs scalar field $\Phi$.

In Fig. 8(top) we present results for spinindependent cross-section for $V^{0}$-nucleon elastic scattering as a function of $M_{V}$ and for representative values of $a$. It is very important to note that Xenon1T experiment excludes DM mass above $4 \mathrm{TeV}$, while from Fig. 8(bottom) one can see that LHC excludes now $1.4 \mathrm{TeV}$ vector DM, while $100 \mathrm{TeV}$ collider will be able to exclude DM mass below $4 \mathrm{TeV}$ and therefore to probe the entire parameter space of the model.

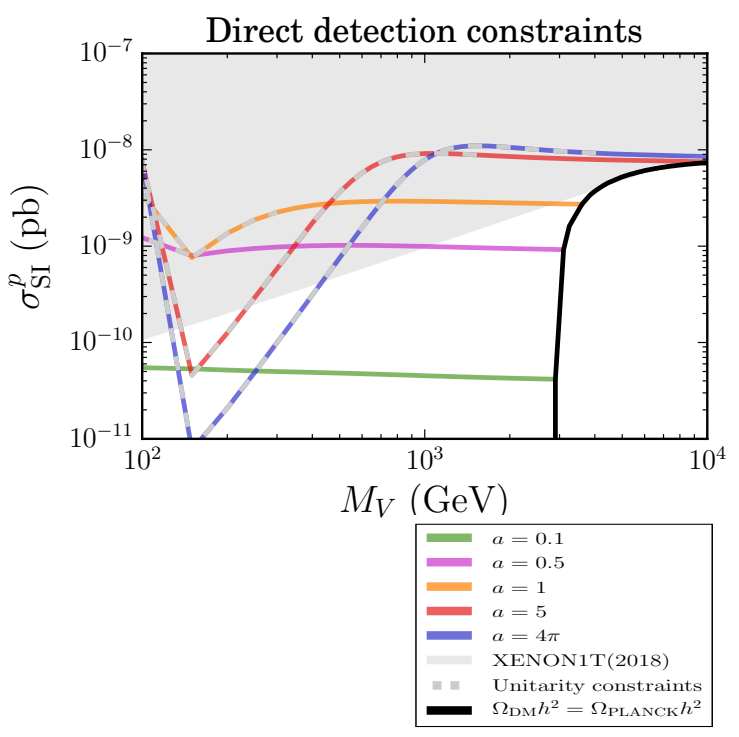

LHC@13, @27TeV and FCC@100 TeV constraints from LLP searches

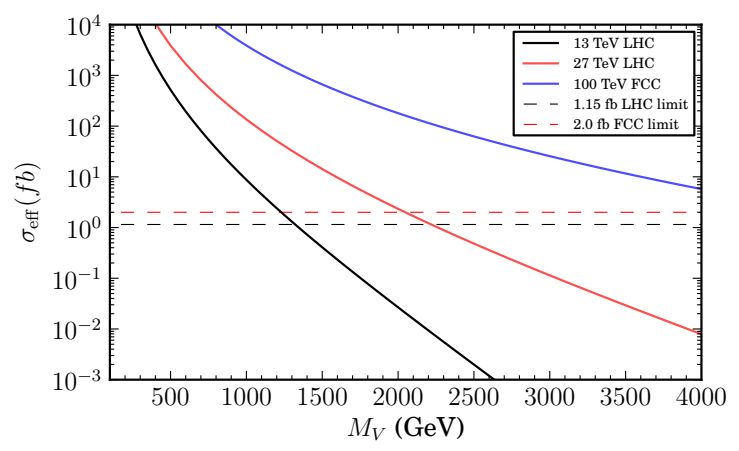

Figure 8: Top: Spin-independent cross-section for $V^{0}$-nucleon elastic scattering as a function of $M_{V}$ and for representative values of $a$. The continuous black curve represents the elastic cross-section computed with the values of $M_{V}$ and $a$ that saturate the measured DM relic density. The grey dashing highlights the parameter space where perturbative unitarity loss. Bottom: the effective cross-sections $\sigma_{\text {eff }}=\sigma\left(p p \rightarrow V^{ \pm} V^{0}\right)+2 \sigma\left(p p \rightarrow V^{+} V^{-}\right)$at leading order for the vector isotriplet model for 13 and $27 \mathrm{TeV}$ LHC energies and for a $100 \mathrm{TeV}$ future collider. The dashed lines corresponds to collider sensitivity. See details in [30]. 
One should also note that in case of i2HDM, DCT signature also allows to substantially enhance LHC potential and probe DM mass upto about $500 \mathrm{GeV}$ [32] which is much higher than $100 \mathrm{GeV}$ - the maximum DM mass which can be probed via mono-jet signature.

\section{Towards DM decoding framework}

There is no framework at the moment which can solve the reverse engineering task - the task of decoding the nature of DM. It is not surprising why - we are all eagerly looking for the signal first of all and busy with the interpreting and exploring of our own models. There is a huge amount of work has been done on the model building, phenomenology and experimental searches as well as on building different tools examples of which has been given above. And there is really huge potential of combining different methods and signatures to probe different models. What is missing is the framework which join all these pieces in one tool which would help us to decode underlying theory, in particular its part related to DM. The task of decoding of the whole underlying theory sounds probably too ambitious to the author, while decoding of its DM part sound more realistic since it contain specific and possibly much smaller piece of the theory. This framework requires the database of models, database of various signatures and set of tools which will be able to effectively explore not only parameter space of each particular model, but also the model parameter space and compare predicted signatures with the observed ones. Such a framework would allow objectively judge about preferred model or set of models which would fit signal best of all. An example of the prototype of such a framework actually already exists in the form of High Energy Physics Model Data Base (HEPMDB) (https://hepmdb.soton.ac.uk) [35], created at Southampton University in 2011. At the moment HEPMDB is created as a webserver accessible to everybody and is able to:

1. collect HEP models for all multipurpose Matrix Element (ME) generators in the form of Feynman rules and parameters written in the format specific for a given package;

2. collect models' sources which can be used to generate HEP models for various ME generators using FeynRules [36] or LanHEP [37];

3. allow users to perform simulations for their own models or models available at HEPMDB using the full power of the High Performance Computing (HPC) IRIDIS cluster standing behind the HEPMDB itself. Connection to HPC cluster is one of the key features of the HEPMDB: it provides a web interface to various ME generators (CalcHEP, Madgraph and Whizard at the moment) which can then also be run directly on the HPC cluster avoiding problems related to installing the actual software, which can sometimes be quite cumbersome;

4. collect simulated events and plot distributions using web interface.

Though the signature database at HEPMDB is at the development stage, users can indicate some essential features of the signatures which model can provide, such as presence of resonance, $\boldsymbol{E}_{\mathrm{T}}^{\mathrm{miss}}$ etc. The next step of development of HEPMDB will include an addition of various packages to event analysis. Probably the most important feature of HEPMDB is that it can be developed by the whole HEP community - any registered user can add his/her own model and signature which would be used for identification of underlying theory when the experimental signal will be observed. 


\section{Conclusions}

In the absence of DM signal we can still do a lot - we can prepare ourselves ot its discovery and identification. $\boldsymbol{E}_{\mathrm{T}}^{\text {miss }}$ shape is quite instrumental in understanding the underlying theory at colliders, while direct and indirect DM searches are very powerful in complementing collider searches especially in the parameter space with large DM mass. We also advocate the usage of new DM signatures such as disappearing charge tracks ones which allows to substantially extend collider exploration of large DM mass. Moreover we would like to stress the crucial role of $100 \mathrm{TeV} p p$ collider which is likely to explore the complete parameter space of thermal DM. We show that collider and noncollider DM searches have a unique power to decode the nature of Dark Matter on the examples of several appealing DM theories. Such complementarity and usage of different signatures would allow us to decode the nature of DM, signals from which we are expecting in the near future. Finally, we advocate the importance of the framework which would combine the experience of HEP community and would allow to identify effectively underlying theory.

\section{Acknowledgements}

Author would like to thank organisers of CORFU2019 workshop for the invitation and very warm hospitality and acknowledges partial support from the STFC grant ST/L000296/1, and Soton-FAPESP grant. AB also thanks the NExT Institute and Royal Society International Exchange grant IE150682.

\section{References}

[1] Planck Collaboration, P. A. R. Ade et. al., Astron. Astrophys. 594 (2016) A13 [1502.01589].

[2] G. R. Blumenthal, S. M. Faber, J. R. Primack and M. J. Rees, Nature 311 (1984) 517-525. [,96(1984)].
[3] J. S. Bullock, T. S. Kolatt, Y. Sigad, R. S. Somerville, A. V. Kravtsov, A. A. Klypin, J. R. Primack and A. Dekel, Mon. Not. Roy. Astron. Soc. 321 (2001) 559-575 [astro-ph/9908159].

[4] ATLAS Collaboration, M. Aaboud et. al., JHEP 01 (2018) 126 [1711. 03301 ].

[5] CMS Collaboration, C. Collaboration.

[6] ATLAS Collaboration, Tech. Rep. ATLAS-CONF-2018-005, CERN, Geneva, Apr, 2018.

[7] ATLAS Collaboration, M. Aaboud et. al., Eur. Phys. J. C77 (2017), no. 6393 [1704.03848].

[8] WMAP Collaboration, G. Hinshaw et. al., Astrophys. J. Suppl. 208 (2013) 19 [1212.5226].

[9] M. W. Goodman and E. Witten, Phys. Rev. D31 (1985) 3059. [,325(1984)].

[10] XENON Collaboration, E. Aprile et. al., Phys. Rev. Lett. 119 (2017), no. 18181301 [1705.06655].

[11] LUX Collaboration, D. S. Akerib et. al., Phys. Rev. Lett. 118 (2017), no. 2021303 [1608.07648].

[12] PandaX-II Collaboration, X. Cui et. al., Phys. Rev. Lett. 119 (2017), no. 18181302 [1708.06917].

[13] T. R. Slatyer in Theoretical Advanced Study Institute in Elementary Particle Physics: Anticipating the Next Discoveries in Particle Physics (TASI 2016) Boulder, CO, USA, June 6-July 1, 2016, 2017. 1710.05137.

[14] Fermi-LAT Collaboration, M. Ackermann et. al., Phys. Rev. Lett. 115 (2015), no. 23 231301 [1503.02641].

[15] VERITAS Collaboration, B. Zitzer, $P o S$ ICRC2015 (2016) 1225 [1509. 01105 ].

[16] Fermi-LAT, MAGIC Collaboration, M. L. Ahnen et. al., JCAP 1602 (2016), no. 02039 [1601.06590].

[17] H.E.S.S. Collaboration, H. Abdallah et. al., Phys. Rev. Lett. 117 (2016), no. 11111301 [1607.08142]. 
[18] H.E.S.S. Collaboration, A. Abramowski et. al., Phys. Rev. Lett. 110 (2013) 041301 [1301.1173].

[19] S. Galli, F. Iocco, G. Bertone and A. Melchiorri, Phys. Rev. D80 (2009) 023505 [0905.0003].

[20] S. Galli, F. Iocco, G. Bertone and A. Melchiorri, Phys. Rev. D84 (2011) 027302 [1106.1528].

[21] J. Goodman, M. Ibe, A. Rajaraman, W. Shepherd, T. M. Tait et. al., Phys.Rev. D82 (2010) 116010 [1008 . 1783].

[22] J. Kumar, D. Marfatia and D. Yaylali, Phys. Rev. D92 (2015), no. 9095027 [1508.04466].

[23] A. Belyaev, L. Panizzi, A. Pukhov and M. Thomas, JHEP 04 (2017) 110 [1610.07545].

[24] XENON Collaboration, E. Aprile et. al., Phys. Rev. Lett. 121 (2018), no. 11111302 [1805.12562].

[25] A. Belyaev, E. Bertuzzo, C. Caniu Barros, O. Eboli, G. Grilli Di Cortona, F. Iocco and A. Pukhov, 1807.03817.

[26] R. J. Hill and M. P. Solon, Phys. Lett. B707 (2012) 539-545 [1111.0016].

[27] M. T. Frandsen, U. Haisch, F. Kahlhoefer, P. Mertsch and K. Schmidt-Hoberg, JCAP 1210 (2012) 033 [1207.3971].
[28] L. Vecchi, 1312.5695.

[29] D. Barducci, A. Belyaev, A. K. M. Bharucha, W. Porod and V. Sanz, JHEP 07 (2015) 066 [1504.02472].

[30] A. Belyaev, G. Cacciapaglia, J. Mckay, D. Marin and A. R. Zerwekh, 1808.10464.

[31] A. Belyaev, T. R. Fernandez Perez Tomei, P. G. Mercadante, C. S. Moon, S. Moretti, S. F. Novaes, L. Panizzi, F. Rojas and M. Thomas, 1809.00933.

[32] A. Belyaev, G. Cacciapaglia, I. P. Ivanov, F. Rojas-Abatte and M. Thomas, Phys. Rev. D97 (2018), no. 3035011 [1612.00511].

[33] R. Mahbubani, P. Schwaller and J. Zurita, JHEP 06 (2017) 119 [1 703 . 0 5327]. [Erratum: JHEP10,061(2017)].

[34] A. R. Zerwekh, Int. J. Mod. Phys. A28 (2013) 1350054 [1207.5233].

[35] M. Bondarenko, A. Belyaev, J. Blandford, L. Basso, E. Boos, V. Bunichev et. al., 1203.1488 .

[36] A. Alloul, N. D. Christensen, C. Degrande, C. Duhr and B. Fuks, Comput. Phys. Commun. 185 (2014) 2250-2300 [1310.1921].

[37] A. Semenov, 1005.1909. 